\title{
Preclinical and Clinical Evidence of Antioxidant Effects of Antidepressant Agents: Implications for the Pathophysiology of Major Depressive Disorder
}

\author{
Guilherme A. Behr,, ${ }^{1,}$ José C. F. Moreira, ${ }^{2}$ and Benicio N. Frey ${ }^{1}$ \\ ${ }^{1}$ Mood Disorders Program and Women's Health Concerns Clinic, Department of Psychiatry and Behavioural Neurosciences, \\ McMaster University, 301 James Street South, Suite F614, Hamilton, ON, Canada L8P 3B6 \\ ${ }^{2}$ Center of Oxidative Stress Research, Professor Tuiskon Dick Department of Biochemistry, Institute of Health Basic Sciences, \\ Federal University of Rio Grande do Sul (UFRGS), Ramiro Barcelos Street, 2600 Anexo, 90035-003 Porto Alegre, RS, Brazil
}

Correspondence should be addressed to Guilherme A. Behr, guibehr@gmail.com

Received 4 February 2012; Accepted 2 March 2012

Academic Editor: Daniel Pens Gelain

Copyright ( $) 2012$ Guilherme A. Behr et al. This is an open access article distributed under the Creative Commons Attribution License, which permits unrestricted use, distribution, and reproduction in any medium, provided the original work is properly cited.

\begin{abstract}
Major depressive disorder (MDD) is a common mental disorder associated with a significant negative impact on quality of life, morbidity/mortality, and cognitive function. Individuals who suffer with MDD display lower serum/plasmatic total antioxidant potentials and reduced brain GSH levels. Also, F2-isoprostanes circulatory levels are increased in MDD subjects and are correlated with the severity of depressive symptoms. Urinary excretion of 8-OHdG seems to be higher in patients with MDD compared to healthy controls. Despite the fact that antidepressant drugs have been used for more than 50 years, their mechanism of action is still not fully understood. This paper examines preclinical (in vitro and animal model) and clinical literature on oxidative/antioxidant effects associated with antidepressant agents and discusses their potential antioxidant-related effects in the treatment of MDD. Substantial data support that MDD seems to be accompanied by elevated levels of oxidative stress and that antidepressant treatments may reduce oxidative stress. These studies suggest that augmentation of antioxidant defences may be one of the mechanisms underlying the neuroprotective effects of antidepressants in the treatment of MDD.
\end{abstract}

\section{Introduction}

Despite the fact that antidepressant drugs have been used for more than 50 years, their mechanism of action is still not fully understood. The hypothesis that antidepressants restore noradrenergic and serotoninergic neurotransmitter systems has been dominant [1]. Recently, a new concept of antidepressants action has been suggested, based on growing evidence demonstrating antioxidant effects of antidepressants in the treatment of major depressive disorder (MDD) (Table 1). This paper examines preclinical (in vitro and animal models) and clinical literature on oxidative/antioxidant effects of antidepressant agents and discusses the relevance of intracellular oxidative pathways in the pathophysiology of MDD.

\section{Oxidative Stress and Antioxidants: Background}

Reactive oxygen species (ROS) are continuously generated in physiological conditions and are effectively controlled/eliminated by intracellular and extracellular antioxidant systems [2]. ROS are products of normal cellular metabolism and are well recognized for their dual role as deleterious and essential compounds, given that ROS can be harmful or beneficial [3]. Beneficial effects of ROS occur at low levels and involve cell signalling and signal transduction [4]. ROS also play an essential role in the human immune system helping killing and eliminating infectious organisms. However, elevated or chronic inflammations are major determinants of disease later in the human lifespan, and ROS 
TABle 1: Antioxidant effects of antidepressant agents: preclinical and clinical studies.

\begin{tabular}{|c|c|c|c|c|}
\hline Antidepressant & $\begin{array}{l}\text { Oxidat } \\
\text { In vitro }\end{array}$ & $\begin{array}{l}\text { ive/Antioxidant-1 } \\
\text { Animal models }\end{array}$ & $\begin{array}{l}\text { related effects } \\
\text { Human data }\end{array}$ & Drug class \\
\hline Amitriptyline & + & + & & TCA \\
\hline Bupropion & & + & & NDRI \\
\hline Citalopram & & & + & SSRI \\
\hline Desipramine & + & & & TCA \\
\hline Duloxetine & & & & SNRI \\
\hline Escitalopram & & + & + & SSRI \\
\hline Fluoxetine & + & + & + & SSRI \\
\hline Fluvoxamine & + & & + & SSRI \\
\hline Imipramine & + & + & & TCA \\
\hline Maprotiline & + & & & TCA \\
\hline Milnacipran & & & + & SNRI \\
\hline Mirtazapine & + & & & NaSSA \\
\hline Moclobemide & & & + & MAOI \\
\hline Nefazodone & & & + & SNDRI \\
\hline Nortriptyline & + & & & TCA \\
\hline Paroxetine & & & + & SSRI \\
\hline Reboxetine & + & & + & NRI \\
\hline Sertraline & & & + & SSRI \\
\hline Tianeptine & & & + & SSRE \\
\hline Trazodone & & & + & SARI \\
\hline Venlafaxine & & + & + & SNRI \\
\hline
\end{tabular}

MAOI: monoamine oxidase inhibitor; NaSSA: noradrenergic and specific serotonergic antidepressant; NDRI: norepinephrine-dopamine reuptake inhibitor; NRI: norepinephrine reuptake inhibitor; SARI: serotonin antagonist and reuptake inhibitor; SNDRI: serotonin-norepinephrine-dopamine reuptake inhibitor; SNRI: serotonin-norepinephrine reuptake inhibitor; SSRE: selective serotonin reuptake enhancer; SSRI: selective serotonin reuptake inhibitor; TCA: tricyclic or tetracyclic antidepressant.

play a critical role in several age-related diseases, particularly cancer, cardiac and neurodegenerative disorders [5]. The major source of ROS in humans is the leakage of superoxide anion $\left(\mathrm{O}_{2}{ }^{--}\right)$from mitochondria during oxidative phosphorylation. Another minor source of ROS is cytoplasmatic, including the $\mathrm{O}_{2}{ }^{--}$generating enzymes such as xanthine oxidase (XO), NADPH oxidases, and cytochromes P450 (CytP450). The main ROS include $\mathrm{O}_{2}{ }^{\cdot-}$, hydrogen peroxide $\left(\mathrm{H}_{2} \mathrm{O}_{2}\right)$, and hydroxyl radical $\left(\mathrm{OH}^{\bullet}\right) . \mathrm{OH}^{\bullet}$ is a strong oxidant formed during Fenton $\left(\mathrm{Fe}^{2+}+\mathrm{H}_{2} \mathrm{O}_{2} \rightarrow \mathrm{Fe}^{3+}+\mathrm{OH}^{\bullet}+\mathrm{OH}^{-}\right)$ and Haber-Weiss $\left(\mathrm{H}_{2} \mathrm{O}_{2}+\mathrm{OH}^{\bullet} \rightarrow \mathrm{H}_{2} \mathrm{O}+\mathrm{O}_{2}{ }^{\bullet-}+\mathrm{H}^{+}\right.$and $\left.\mathrm{H}_{2} \mathrm{O}_{2}+\mathrm{O}_{2}{ }^{\bullet-} \rightarrow \mathrm{O}_{2}+\mathrm{OH}^{-}+\mathrm{OH}^{\bullet}\right)$ reactions. Additionally, some nitrogen species can be potentially dangerous to the cell, such as peroxynitrite (ONOO-), which is formed in a rapid reaction between $\mathrm{O}_{2}{ }^{--}$and nitric oxide (NO) [3].

The main enzymatic antioxidant defences include superoxide dismutase (SOD), catalase (CAT), and glutathione peroxidase (GPx). SOD enzymes are highly efficient in the catalytic dismutation of $\mathrm{O}_{2}{ }^{--}$and generation of $\mathrm{H}_{2} \mathrm{O}_{2}$ which, in turn, can be removed by two types of enzymes - the catalases (CAT) and peroxidases (e.g., GPx). Importantly, the activity of GPx is closely dependent on glutathione reductase (GR), glutathione tripeptide (GSH), and others cofactors.
Moreover, virtually all cells contain nonenzymatic defenses, like GSH, vitamins C (ascorbate) and E (alpha-tocopherol), and metal-binding and related protective proteins [37].

The term "oxidative stress" has been defined as an imbalance between the generation of ROS and antioxidant defenses, favouring the former [3]. In situations of oxidative stress, several biomolecules (e.g., lipid membrane, proteins, and DNA) can be damaged. Because ROS have extremely short half-lives, they are difficult to measure. Therefore, most studies measure products of the damage induced by oxidative stress. For instance, malondialdehyde (MDA) is one of the low-molecular-weight end products formed via the decomposition of primary and secondary lipid peroxidation products [38]. MDA and other thiobarbituric reactive substances (TBARS) condense with two equivalents of thiobarbituric acid that can be assayed spectrophotometrically [39]. Another compound commonly used as a biomarker of oxidative stress is 4-Hydroxynonenal (4-HNE). 4 -HNE is generated in the oxidation of lipids containing polyunsaturated omega- 6 acyl groups, such as arachidonic or linoleic groups, and the corresponding fatty acids [40]. Perhaps the most accurate markers of lipid peroxidation are the isoprostanes (i.e., F2-isoprostanes). Isoprostanes are prostaglandin-like compounds formed in vivo from the free radical-catalyzed peroxidation of essential fatty acids (primarily arachidonic acid) [41]. Proteins are possibly the most immediate targets of cellular oxidative damage. Carbonyl groups (aldehydes and ketones) are produced in protein side chains (especially of Pro, Arg, Lys, and Thr) when they are oxidized, which can be measured by specific techniques [42]. Another method to evaluate levels of oxidation/reduction content in biological samples is the total reduced thiol $(-\mathrm{SH})$ quantification [43]. ROS can also attack and damage the DNA, thereby generating 8-hydroxydeoxyguanosine (8oxodG) and 8-hydroxyguanosine (8-oxoG) [37].

Additionally, total antioxidant potentials can be measured using various methods such as TAC, total antioxidant capacity; TRAP, total-radical nonenzymatic antioxidant potential; OSI, oxidative stress index; TOS, total oxidant status. Low total antioxidant capacity could be indicative of oxidative stress or increased susceptibility to oxidative damage [44].

\section{Oxidative Stress in Major Depressive Disorder}

MDD is one of the most common mental disorders among humans and it is associated with a significant negative impact on quality of life, morbidity/mortality, and cognitive function. The pathophysiology of depression is multifactorial and includes changes in brain monoaminergic transmission (e.g., 5-HT, NE, DA), abnormalities in neurotransmitter receptors function (e.g., AC-cAMP pathway), reduced neurotrophic factors (e.g., BDNF), dysregulation of HPA axis (cortisol), increased proinflammatory cytokines (e.g., IL-6, TNF- $\alpha$, NF$\kappa \mathrm{B})$, increased NO (e.g., L-arginine-NO-cGMP pathway), and increased oxidative stress (e.g., lipid and DNA damage) [45-47]. 
Individuals who suffer with MDD display lower serum/ plasmatic total antioxidant potentials $[28,32,48]$ and reduced brain GSH levels [31] as compared to matched controls. Plasmatic coenzyme Q10 (CoQ10), a strong antioxidant and a key molecule in the mitochondrial electron transport chain, is significantly lower in major depressive patients [34], which indicates lower antioxidant defenses against oxidative stress. Moreover, increased serum XO levels observed in MDD subjects suggest increased systemic ROS production [29]. XO is a widely distributed enzyme involved in later stages of purine catabolism, which catalyzes the oxidation of hypoxanthine to xanthine and of xanthine to uric acid, both reactions with potential to generate $\mathrm{O}_{2}{ }^{--}$and $\mathrm{H}_{2} \mathrm{O}_{2}$ [49]. A recent post-mortem study found increased $\mathrm{XO}$ activity in the thalamus and putamen patients with recurrent MDD [35].

Dimopoulos et al. (2008) have found that F2-isoprostanes (F2-iso) circulatory levels were increased in major depressive patients and were significantly correlated with the severity of depressive symptoms [50]. The presence of detectable quantities of F2-iso in human biological fluids implies ongoing lipid peroxidation [51]. Furthermore, urinary excretion of 8-OHdG, a marker of oxidative damage to DNA, was found to be higher in patients with MDD than healthy controls [52].

\section{Antioxidant Effects of Antidepressants}

4.1. Studies In Vitro. The main findings of in vitro assays using rat mitochondria and cell culture protocols are depicted in Table 2. Kolla et al. (2005) have demonstrated that pretreatment with amitriptyline and fluoxetine protects against oxidative stress-induced damage in rat pheochromocytoma (PC12) cells. Both drugs attenuated the decrease in cell viability induced by $\mathrm{H}_{2} \mathrm{O}_{2}$ in PC12 cells. Also, pretreatment with amitriptyline and fluoxetine was associated with increased SOD activity, and no signs of cell death were observed in the treated cells [10]. In another study, pretreatment with imipramine, fluvoxamine, or reboxetine inhibited NO production in a dose-dependent manner in an activated microglia cell culture protocol [11]. The authors suggested that these antidepressant drugs have inhibitory effects on IFN- $\gamma$-activated microglia and that these effects are, at least in part, mediated by cAMP-dependent PKA pathway.

Schmidt et al. (2008) examined the effects of desipramine, imipramine, maprotiline and mirtazapine on mRNA levels of various antioxidant enzymes in human monocytic U-937 cells [12]. In this study, short-term treatment with these drugs decreased mRNA levels of SOD and CAT. However, long-term treatment increased mRNA levels of SOD, GST, and GR. These results suggest that the effects of these antidepressants on the expression of antioxidant enzymes are dependent on the duration of the treatment regimen. Zhang et al. (2008) showed for nortriptyline some antioxidant effects using isolated rat liver mitochondria or PCN cell culture. Nortriptyline was able to inhibit loss of mitochondrial membrane potential and the activation of caspase 3 in isolated rat liver mitochondria and decrease cell death induced by oxygen/glucose deprivation on PCN cells [9].

The antioxidant effects of fluoxetine on isolated rat brain and liver mitochondria have been extensively studied. Curti et al. (1999) reported that fluoxetine can indirectly and nonspecifically affect electron transport and $\mathrm{F}_{1} \mathrm{~F}_{0}$-ATPase activity, thereby inhibiting oxidative phosphorylation in rat brain [6]. Two studies that evaluated the effects of fluoxetine in rat liver mitochondria revealed mixed results. Souza et al. (1994) reported that fluoxetine may be potentially hepatotoxic at high doses [7]. However, Nahon et al. (2005) demonstrated that fluoxetine was able to inhibit the opening of the mitochondrial permeability transition (MPT) pore, the release of cytochrome c (cytC) and protected against staurosporine-induced apoptotic cell death [8]. An important difference between these two studies is the fact that Souza et al. used isolated liver mitochondria and tested fluoxetine at different concentrations in order to establish potential toxic doses. On the other hand, Nahon et al. challenged isolated mitochondria against staurosporine-induced damage and showed protective effects of fluoxetine in this model.

In summary, studies in vitro not only revealed antioxidant-related effects for antidepressant drugs, but also some potential prooxidant effects specifically in rat liver with fluoxetine at higher dosages. Cell culture and isolated tissues studies are used extensively in research and drug development; however, these techniques have some limitations and studies using live organisms (i.e., rodents) are necessary to better evaluate safety as well as behavioural effects.

4.2. Animal Models. Several animal model protocols have been used to investigate oxidative/antioxidant-related effects of antidepressant drugs. Table 3 summarizes the studies conducted with acute and chronic antidepressant treatments in control and stressed animals.

Réus et al. (2010) reported increased SOD and CAT activity and decreased lipid and protein damage in male rat prefrontal cortex and hippocampus after both acute and chronic treatment with imipramine [17]. Additionally, imipramine treatment increased brain creatine kinase and increased activity of mitochondrial respiratory chain complexes $[18,53]$. Katyare and Rajan (1995) showed that longterm administration of imipramine to female rats resulted in significant stimulation of the states 3 and 4 respiration rates. This effect was evident within a week of imipramine administration and was sustained through the second week of treatment [20]. These results suggest that imipramine treatment may induce changes in substrate oxidation pattern, increase rate of ATP synthesis, and can potentially increase mitochondrial ROS production.

$\mathrm{Xu}$ et al. (2003) examined dose-dependent effects of amitriptyline and venlafaxine on neuroprotective proteins in male rats. In this study, low dose $(5 \mathrm{mg} / \mathrm{kg})$ of amitriptyline and venlafaxine increased the intensity of BDNF immunostaining in hippocampal pyramidal neurons and the intensity of Bcl-2 immunostaining in hippocampal mossy fibers, but did not alter the Cu/Zn-SOD immunoreactivity. High 


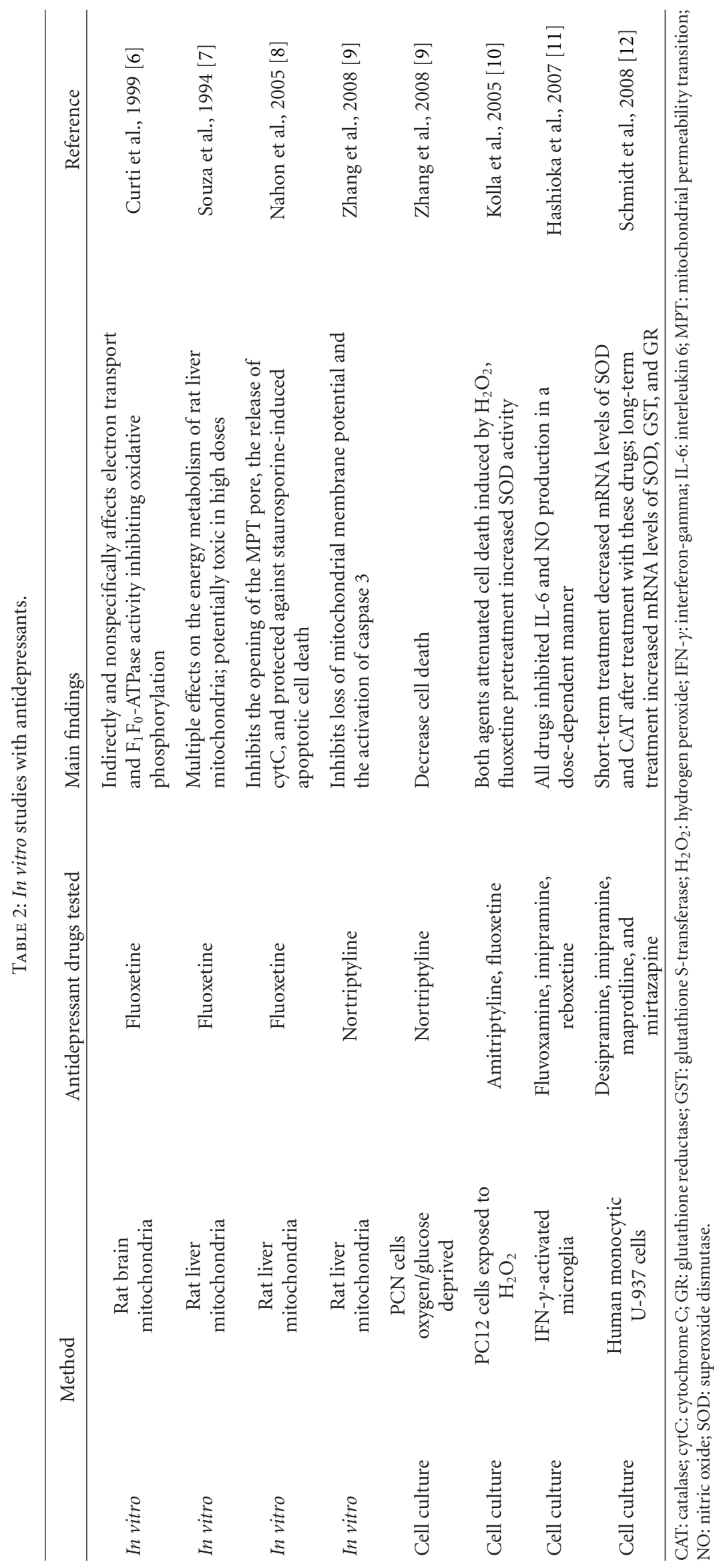




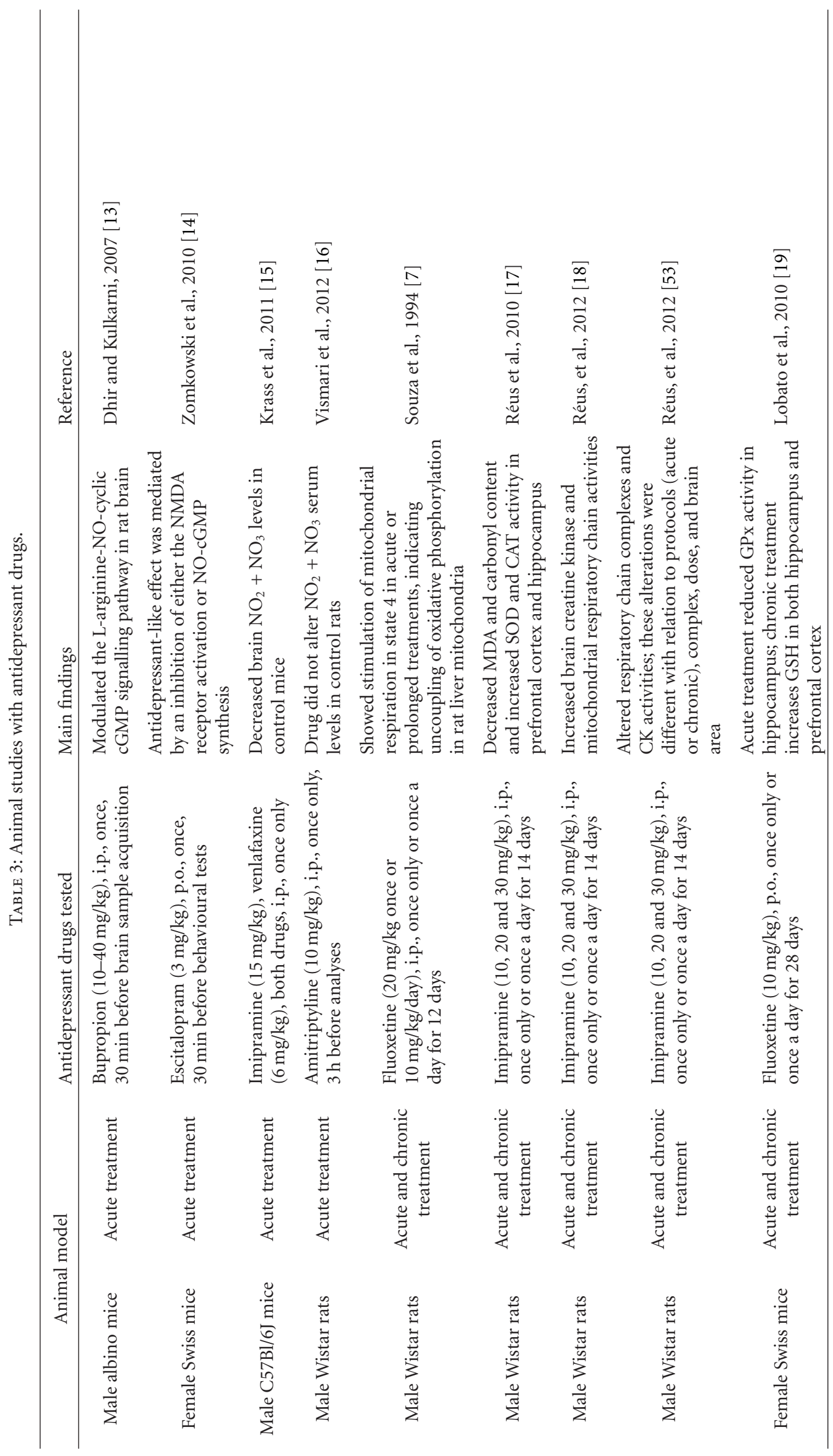




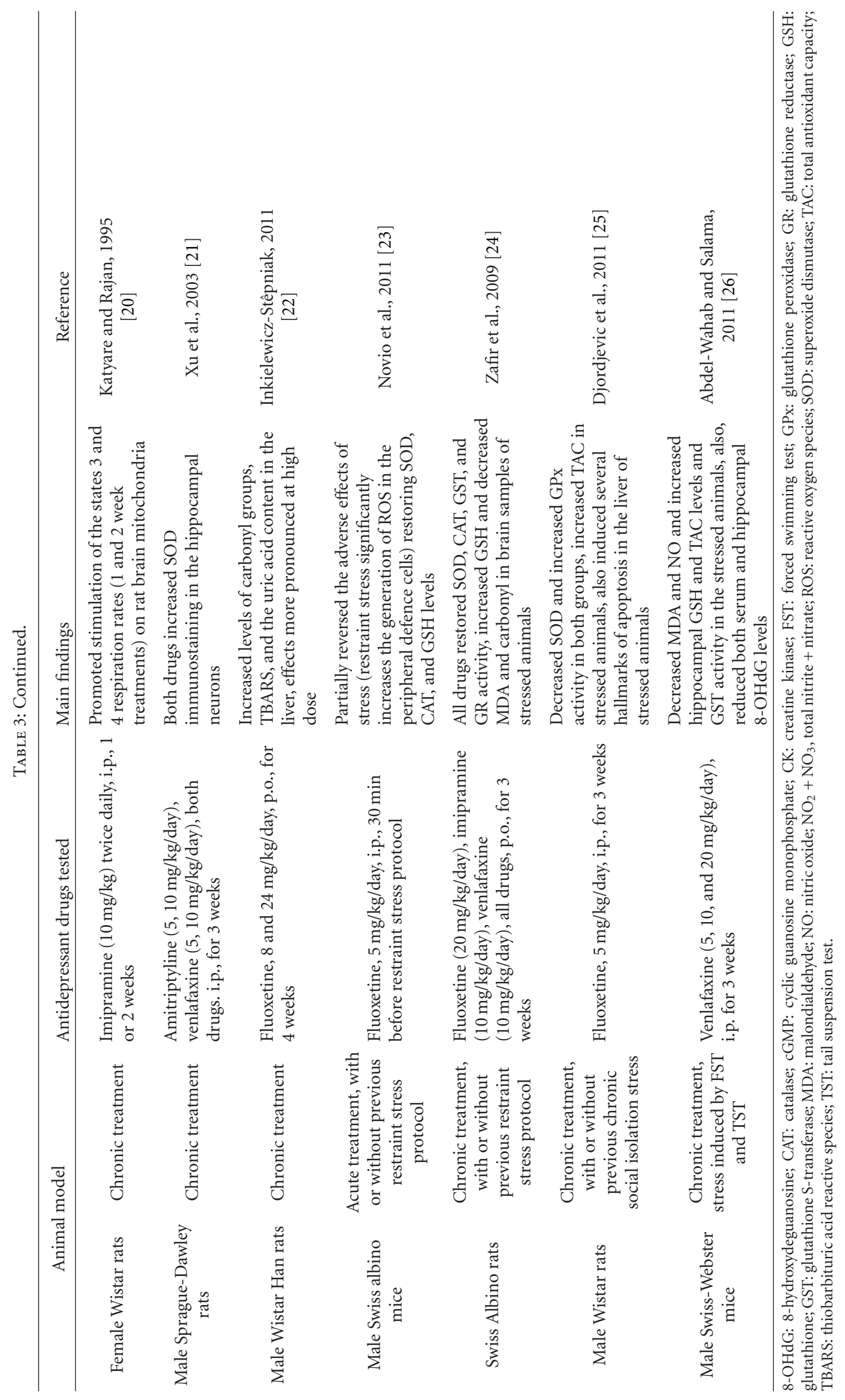


dose $(10 \mathrm{mg} / \mathrm{kg})$ of venlafaxine, however, decreased the intensity of BDNF immunostaining in all subareas of the hippocampus and increased the intensity of $\mathrm{Cu} / \mathrm{Zn}-\mathrm{SOD}$ immunostaining in the dentate granular cell layer [21]. More recently, Abdel-Wahab and Salama (2011) showed that long-term venlafaxine treatment at effective antidepressant dosages can protect against stress-induced oxidative cellular and DNA damage in male mice. At all doses tested, venlafaxine decreased MDA and total nitrite levels, increased total antioxidant potential and GSH content, and restored GST activity in hippocampus of stressed animals. Venlafaxine also promoted increased total antioxidant potential and GSH levels in the control, nonstressed group. Finally, this treatment was able to reduce serum and hippocampal levels of 8-OHdG (a marker of DNA damage) in stressed animals [26] showing potential antioxidant effects related to these antidepressant agents.

The effects of chronic (one month) fluoxetine treatment on lipid and protein oxidative damage, uric acid concentration in the liver and the activity of transaminases and transferases in the serum have been investigated in male rats. Chronic fluoxetine treatment increased the levels of TBARS, carbonyl groups, and the uric acid content in the liver. The activities of alanine transaminase (ALT), aspartate transaminase (AST), and GST were increased in the serum. The overall effects are more pronounced in the higher dose (24 versus $8 \mathrm{mg} / \mathrm{kg}$ ) [22]. More recently, Djordjevic et al. (2011) showed altered antioxidant status and increased apoptotic signalling in male rat liver after 21 days of fluoxetine treatment. Control animals and stressed animals displayed decreased activity of SOD and increased activity of GPx. In addition, in both experimental groups, fluoxetine altered several markers of apoptosis in the liver, including decreased $\mathrm{Bcl}-2$ expression and increased DNA fragmentation [25]. These effects seemed to be associated with liver toxicity induced by high-dose fluoxetine treatment in rats.

Novio et al. (2011) investigated the effects of fluoxetine on intracellular redox status in peripheral blood cells obtained from male mice exposed to restraint stress. They found that restraint stress significantly increased the generation of ROS in the peripheral blood and that acute treatment with fluoxetine partially reversed this effect, possibly through normalization of SOD and CAT activity and GSH content [23]. Using a depression-like rat model, Zafir et al. (2009) examined antioxidant effects of fluoxetine and venlafaxine in the rat brain. The results evidenced a significant recovery in the activities of SOD, CAT, GST, GR, and GSH levels by these antidepressants after restraint stress. Also, fluoxetine and venlafaxine treatment prevented lipid and protein oxidative damage induced by stress [24]. In another study, acute fluoxetine treatment reduced GPx activity in the hippocampus, whereas chronic treatment increased GSH in both hippocampus and prefrontal cortex of female mice [19].

Recent data support that some antidepressants are able to modulate NO synthesis and nitrosative stress-associated signalling cascades. Dhir and Kulkarni (2007) tested different dosages of bupropion in male rats. The antidepressant-like effect of bupropion was prevented by pretreatment with Larginine (a substrate of nitric oxide synthase, NOS). Pretreatment with 7-nitroindazole (a specific neuronal NO synthase, nNOS inhibitor) potentiated bupropion's effects. In addition, treatment with methylene blue (a direct inhibitor of NOS and soluble guanylate cyclase, sGC) potentiated the effect of the drug in the forced swim test [13]. This study suggests that bupropion possesses antidepressant-like activities in different animal models possibly through dopaminergic and L-arginine-NO-cyclic guanosine monophosphate (cGMP) signaling pathways. This is consistent with a study by Zomkowski et al. (2010) showing similar effects with escitalopram in female mice. The antidepressant-like effect of escitalopram in the forced swim test (FST) was prevented by pretreatment with $\mathrm{N}$-methyl-D-aspartic acid (NMDA), L-arginine, and sildenafil (a phosphodiesterase inhibitor). Also, the administration of 7-nitroindazole, methylene blue or ODQ (i.c.v., a soluble sGC inhibitor) in combination with escitalopram reduced the immobility time in the FST. This study highlights the role of NMDA receptors and Larginine-NO-cGMP pathway in the mechanism of action of antidepressant agents [14]. Recently, Krass et al. (2011) reported that imipramine decreased brain nitrite + nitrate $\left(\mathrm{NO}_{2}+\mathrm{NO}_{3}\right)$ levels, a marker of nitrosative stress, in male rat brain. This result supports the idea that antidepressants are able to inhibit NO synthesis in the rat brain [16], an effect that could be mechanistically related to the ability of L-arginine to counteract their antidepressant-like effects [15]. In summary, studies in animal models suggest that antidepressant agents modulate antioxidant enzyme activities and decrease oxidative stress markers on liver, brain, and peripheral tissues. In addition, there is a clear association between high dosages of antidepressants and increased hepatic oxidative stress. However, a major limitation of the studies above mentioned is that not all studies measured oxidative stress markers (i.e., MDA, carbonyl); therefore, these prooxidant effects need further investigation.

Consistent with the above-mentioned studies, changes in the blood/brain antioxidant profile have been associated with changes in depressive-like behaviour. More specifically, it has been demonstrated that some classic antioxidants induce antidepressant-like effects in rodents. In one study, treatment with Ginkgo biloba extract $(10 \mathrm{mg} / \mathrm{kg})$ reduced recorded immobility time in the forced swimming test (FST) to the same extent as imipramine (39\% versus 38\%). No differences in locomotor activity were observed, suggesting a selective antidepressant-like effect. This antidepressantlike effect of Ginkgo biloba extract was associated with a reduction in lipid peroxidation and superoxide radical production (as indicated by a downregulation of SOD activity) [54]. In rats displaying depressive-like behaviour induced by chronic mild stress, administration of liquiritin, an antioxidant derived from Glycyrrhiza uralensis, decreased immobility time, increased sucrose consumption, increased SOD activity, and attenuated MDA production in the peripheral blood [55]. These findings are further corroborated by a study showing that Ebselen (2-phenyl-1,2-benzisoselenazol$3[2 \mathrm{H}]$-one), a substance that mimics the activity of the antioxidant enzyme GPx [56], decreased immobility time 
in rodents, an effect that was dependent on its interaction with the noradrenergic and dopaminergic systems [57]. Additionally, alpha-tocopherol (vitamin E) administration produced antidepressant-like effects in animal models of depression. Along with antidepressant-like effects, longterm treatment with alpha-tocopherol enhanced antioxidant defences in the mouse hippocampus and prefrontal cortex, two structures closely implicated in the pathophysiology of depression [19].

4.3. Post-Mortem Studies. A number of post-mortem studies reported altered oxidative stress parameters in individuals with MDD (Table 4). Michel et al. (2010) showed increased $\mathrm{XO}$ activity in the thalamus and putamen of seven individuals with an ante-mortem diagnosis of recurrent MDD (age range $=61-93$ y.o.). Four of these subjects received SSRI and one was medicated with clomipramine in the 6 months before death, while two of them were not antidepressant treatments [35]. These results suggest increased ROS production in brain samples of depressive patients due to increased $\mathrm{XO}$ activity. Two recent studies showed reduced oxidized and total GSH in the prefrontal cortex of MDD subjects as compared to controls $[31,36]$. In addition, GPx levels were reduced in MDD subjects [31]. Because 10 in 14 patients have taken antidepressants at time of death, we can speculate that antidepressants had limited or no effects on GSH and GPx levels. In a subsequent study with the same cohort, GST levels were also reduced in MDD patients and no effects of antidepressant treatment were observed [36].

In summary, while some changes in antioxidant enzymes have been observed in MDD, these post-mortem studies are not conclusive mostly because of small sample sizes, lack of control groups, and lack of relevant information (i.e., treatment duration, specific drugs used).

\section{Clinical Data: Human Studies}

In the last decade, an increasing number of studies have addressed the potential effects of antidepressant treatments on oxidative stress and antioxidant potential in humans (Table 4). Corroborating with animal data, the majority of these studies revealed that antidepressant agents possess antioxidant properties when used in the treatment of MDD. Increased serum SOD and MDA levels have been found in a cohort of 62 major depressive patients (age $43.8 \pm 12.9$, mean $\pm \mathrm{SD} ; 34 / 28$, female/male ratio) [27]. In another study, plasmatic vitC levels were reduced in patients with MDD compared with age- and sex-matched healthy volunteers ( $n=40)$. Oxidative stress markers (SOD, vitC, lipid peroxidation) were reversed after 4 weeks of treatment with fluoxetine (20 mg/day, $n=32)$ and citalopram $(20 \mathrm{mg} /$ day, $n=30)$. Notably, these antioxidant effects were persistent after 12 weeks of treatment [27].

Bilici et al. (2001) reported increased oxidative stress in major depressive patients $(n=32)$, indexed by higher antioxidant enzyme activities (erythrocyte SOD, GPx, and plasmatic GR) and MDA levels (erythrocyte and plasmatic). After treatment with four different SSRIs drugs (fluoxetine $20 \mathrm{mg} /$ day, $n=7$; sertraline $50 \mathrm{mg} /$ day, $n=13$; fluvoxamine
$100 \mathrm{mg} /$ day, $n=5$; or citalopram $20 \mathrm{mg} /$ day, $n=5$ ), for 12 weeks, antioxidant enzyme activities (plasmatic GPx) and MDA levels (plasma and erythrocyte) were restored to control levels. Plasmatic GR and erythrocyte SOD were also significantly decreased in MD patients after 12-week antidepressant treatment [30]. In another study, a group of $50 \mathrm{MDD}$ patients (age $36.7 \pm 5.2 ; 22 / 28 \mathrm{~F} / \mathrm{M}$ ratio) who had achieved remission from their first episode of depression after 3 months of treatment with $20 \mathrm{mg}$ of fluoxetine were tested before and after remission [48]. Before treatment, MDD patients displayed increased erythrocyte SOD and CAT activities, increased MDA levels, and decreased plasmatic total antioxidant status (TAS) level. After three months of fluoxetine treatment, MDA levels were normalized [48]. Decreased serum SOD and increased XO were found in 20 individuals with MDD (age range 17-62 years, 19/17 F/M ratio) [29]. Although increased XO levels indicate increased free radical production, no difference was observed in serum total nitrite levels (a marker of nitrosative stress, possible associated to ONOO-) between control and MDD patients before treatment. Also, the authors did not find a significant relationship between the duration of illness and SOD, XO activities, or nitrite levels in this cohort. Treatment with citalopram (20 mg/day, $n=10)$, fluoxetine $(20 \mathrm{mg} /$ day, $n=11)$, fluvoxamine (150 mg/day, $n=7)$, or sertraline ( $50 \mathrm{mg} /$ day, $n=8$ ) for 8 weeks increased SOD activity whereas decreased XO levels suggesting that normalization of these enzymes was associated with symptomatic improvement [29].

Cumurcu et al. (2009) investigated whether 3 different total antioxidant parameters (TAC, TOS, and OSI) were associated with MDD and evaluated the impact of antidepressant treatment on these oxidative/antioxidant parameters in a cohort of 57 major depressive patients (age 35.5 \pm 12.1 , 46/11 $\mathrm{F} / \mathrm{M}$ ratio). TOS and OSI were higher and TAC level was lower in the MDD group compared with controls $(n=$ 40). Furthermore, the authors found a positive correlation between the severity of the disease and serum TOS and OSI $(r=0.58$, and $r=0.63$, resp.). Also, a negative correlation was found between the severity of the disease and serum TAC $(r=-0.553)$ at the pretreatment stage. After 3 months of treatment with escitalopram, $10-20 \mathrm{mg} /$ day, $n=10$; paroxetine, $20-40 \mathrm{mg} /$ day, $n=20$; or sertraline, 50-100 mg/day, $n=27$, TOS and OSI were decreased and TAC was increased compared with pretreatment values [32]. These further suggest that recovery from a major depressive episode may be associated with normalization of antioxidant potential induced by antidepressants.

More recently, a 24-week follow-up study evaluated the effects of long-term antidepressant treatment on oxidative/antioxidant status in a cohort of $50 \mathrm{MDD}$ subjects (age $33.1 \pm 10.0,39 / 11 \mathrm{~F} / \mathrm{M}$ ratio) [33]. Antidepressant treatments included venlafaxine $(125 \pm 43.3 \mathrm{mg} /$ day, $n=$ 21), milnacipran ( $100 \mathrm{mg} /$ day, $n=2)$, paroxetine $25 \pm$ $7.6 \mathrm{mg} /$ day, $n=8$, escitalopram $16.3 \pm 5.2 \mathrm{mg} /$ day, $n=$ 8 , sertraline $80 \pm 27.4 \mathrm{mg} /$ day, $n=5$, citalopram $33.3 \pm$ $11.5 \mathrm{mg} /$ day, $n=3$, fluoxetine $20 \mathrm{mg} /$ day, $n=1$, tianeptine $37.5 \mathrm{mg} /$ day, and moclobemide $600 \mathrm{mg}$ /day. Plasmatic MDA, serum oxidized LDL (OxLDL) levels, and erythrocyte SOD 


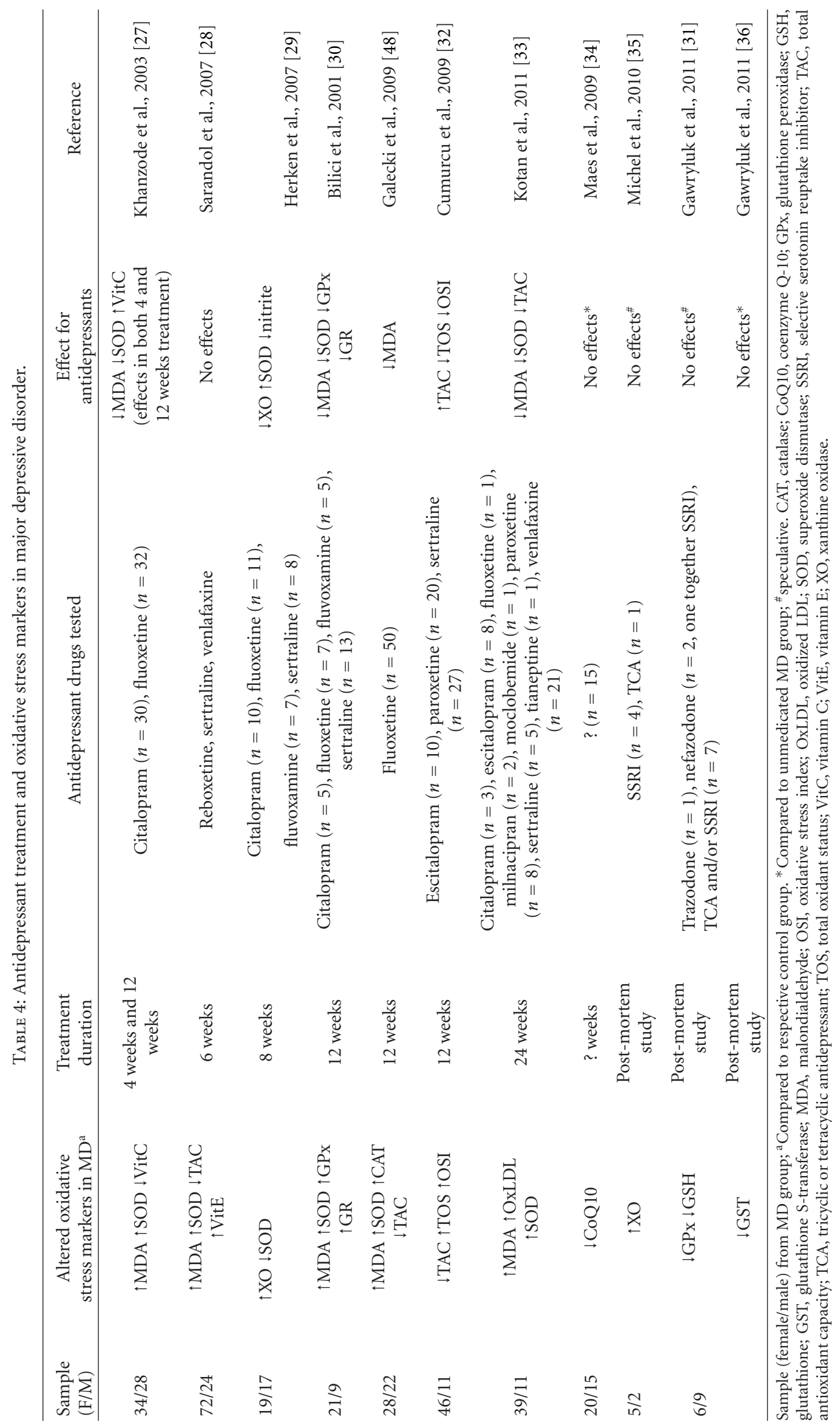


activity were increased in MDD patients before treatment, and MDA levels were positively correlated with the severity of MDD. After 24-weeks of treatment, MDA and SOD levels decreased. However, TAC was also found decreased after 24-week treatment with antidepressants, indicating that the oxidative stress observed in depressed patients was partly improved during 24 weeks of antidepressant treatment. Patients on venlafaxine were also compared with patients on SSRIs in the aspect of oxidative stress parameters in the follow-up period, but no significant differences were found [33].

Sarandol et al. (2007) found that MDD was accompanied by increased peripheral oxidative stress; however, short-term antidepressant treatment (6 weeks) did not alter oxidative/antioxidant systems in a cohort of $96 \mathrm{MDD}$ patients (age $40 \pm 11,72 / 24 \mathrm{~F} / \mathrm{M}$ ratio). In this study, MDD patients had increased plasmatic MDA levels and increased susceptibility of red blood cells (RBCs) to oxidation. Also, SOD activity was increased in patients with MDD, and there was a positive correlation between the severity of depressive symptoms and SOD activity $(r=0.419)$. After 6 weeks of treatment with venlafaxine $75-150 \mathrm{mg} /$ day, sertraline $50 \mathrm{mg} /$ day, or reboxetine $4-8 \mathrm{mg} / \mathrm{day}$, these oxidative parameters were not altered [28].

Maes et al. (2009) investigated plasma concentrations of CoQ10 in 35 depressed patients (age $42.1 \pm 10.5,20 / 15 \mathrm{~F} / \mathrm{M}$ ratio) and 22 sex-, age-matched controls. Plasmatic CoQ10 was lower in depressed patients than controls. However, there was no correlation between plasma CoQ10 and the severity of illness or the number of depressive episodes. During the study, part of the depressed patients were on antidepressant treatment at the time of blood sampling $(n=15)$, while the remaining were unmedicated $(n=20)$. There were no differences in plasma CoQ10 between depressed patients who were taking antidepressants and those without [34].

\section{Concluding Remarks}

This paper examined preclinical (in vitro and animal models) and clinical literature on oxidative/antioxidant effects of antidepressant agents. Overall, most animal and human data support that antidepressant drugs exert antioxidant effects during treatment for MDD.

In vitro and animal studies also suggest that some antidepressants may be prooxidant at high doses. The antioxidant effects of antidepressant drugs seem to vary depending on the dose, treatment regimen, and duration. Notably, a number of clinical trials revealed that treatment with antidepressants can reverse the increased oxidative stress observed in individuals with MDD. Short-term treatments (4 to 8 weeks) do not seem to alter antioxidant/oxidative parameters in MDD patients, whereas longer treatments (12 to 24 weeks) seem to induce robust antioxidant effects.

Overall, the literature reviewed does not support differences in antioxidant potential between different antidepressant agents/classes. However, many of these studies were short in duration and likely underpowered to address the question of differences in antioxidant potential amongst particular drugs and larger studies are warranted.

Brain imaging studies have suggested that MDD may be associated with decreased volumes of various brain regions [58-60]. For instance, MDD subjects have smaller normalized frontal lobe volumes when compared with the nondepressed controls after controlling for age, gender and "total cumulative illness rating scale score" [61]. Presence of temporal lobe atrophy and moderate-to-severe white matter lesions can predict occurrence of major depression during a 5-year followup in a population-based sample of elderly [62]. Considering that the presence of oxidative (and nitrosative) stress may cause neurodegeneration and reduced neurogenesis $[63,64]$, the relationship between oxidative stress and changes in brain structure and function in MDD is a promising area for future studies.

An important issue in biomarker research is the fact that peripheral markers may not necessarily correlate with changes in the central nervous system. For instance, Teyssier et al. (2011) demonstrated that the expression of oxidative stress-response genes was not altered in the prefrontal cortex of individuals with MDD. They concluded that the pathogenic role of oxidative stress in the neurobiology of depression could not be inferred from alterations in the periphery [65]. However, in this post-mortem study all of the patients had received antidepressant treatment, which may have normalized oxidative stress parameters. Furthermore, there is also evidence suggesting that BDNF, oxidative stress, and inflammation tend to be abnormal among individuals with multiple mood episodes and correlate with length of illness [51, 66, 67]. Peripheral biomarkers detected during acute mood episodes could in fact constitute markers of disease activity [68]. Studies of peripheral biomarkers in large randomized, placebo-controlled trials will ultimately confirm whether or not normalization of oxidative stress parameters is associated with treatment response.

In conclusion, there is increasing body of evidence supporting that MDD may be associated with changes in oxidative stress markers and that antidepressant agents (especially long-term treatment) may increase antioxidant defences. It is possible that augmentation of antioxidant defences may be one of the mechanisms underlying the neuroprotective effects of antidepressants observed in the treatment of MDD.

\section{Acknowledgments}

G. A. Behr is recipient of Capes scholarship-Proc. no. BEX 5383/10-2.

\section{References}

[1] E. Castrén, "Is mood chemistry?" Nature Reviews Neuroscience, vol. 6, no. 3, pp. 241-246, 2005.

[2] J. A. Imlay, "Cellular defenses against superoxide and hydrogen peroxide," Annual Review of Biochemistry, vol. 77, pp. 755-776, 2008.

[3] B. Harwell, "Biochemistry of oxidative stress," Biochemical Society Transactions, vol. 35, no. 5, pp. 1147-1150, 2007. 
[4] M. Valko, D. Leibfritz, J. Moncol, M. T. D. Cronin, M. Mazur, and J. Telser, "Free radicals and antioxidants in normal physiological functions and human disease," International Journal of Biochemistry and Cell Biology, vol. 39, no. 1, pp. 4484, 2007.

[5] B. Halliwell, "Oxidative stress and neurodegeneration: where are we now?" Journal of Neurochemistry, vol. 97, no. 6, pp. 1634-1658, 2006.

[6] C. Curti, F. E. Mingatto, A. C. M. Polizello, L. O. Galastri, S. A. Uyemura, and A. C. Santos, "Fluoxetine interacts with the lipid bilayer of the inner membrane in isolated rat brain mitochondria, inhibiting electron transport and F1F0-ATPase activity," Molecular and Cellular Biochemistry, vol. 199, no. 1-2, pp. 103-109, 1999.

[7] M. E. J. Souza, A. C. M. Polizello, S. A. Uyemura, O. CastroSilva, and C. Curti, "Effect of fluoxetine on rat liver mitochondria," Biochemical Pharmacology, vol. 48, no. 3, pp. 535-541, 1994.

[8] E. Nahon, A. Israelson, S. Abu-Hamad, and V. ShoshanBarmatz, "Fluoxetine (Prozac) interaction with the mitochondrial voltage-dependent anion channel and protection against apoptotic cell death," FEBS Letters, vol. 579, no. 22, pp. 51055110, 2005.

[9] W. H. Zhang, H. Wang, X. Wang et al., "Nortriptyline protects mitochondria and reduces cerebral ischemia/hypoxia injury," Stroke, vol. 39, no. 2, pp. 455-462, 2008.

[10] N. Kolla, Z. Wei, J. S. Richardson, and X. M. Li, "Amitriptyline and fluoxetine protect PC12 cells from cell death induced by hydrogen peroxide," Journal of Psychiatry and Neuroscience, vol. 30, no. 3, pp. 196-201, 2005.

[11] S. Hashioka, A. Klegeris, A. Monji et al., "Antidepressants inhibit interferon- $\gamma$-induced microglial production of IL-6 and nitric oxide," Experimental Neurology, vol. 206, no. 1, pp. 33-42, 2007.

[12] A. J. Schmidt, P. Heiser, U. M. Hemmeter, J. C. Krieg, and H. Vedder, "Effects of antidepressants on mRNA levels of antioxidant enzymes in human monocytic U-937 cells," Progress in Neuro-Psychopharmacology and Biological Psychiatry, vol. 32, no. 6, pp. 1567-1573, 2008.

[13] A. Dhir and S. K. Kulkarni, "Involvement of nitric oxide (NO) signaling pathway in the antidepressant action of bupropion, a dopamine reuptake inhibitor," European Journal of Pharmacology, vol. 568, no. 1-3, pp. 177-185, 2007.

[14] A. D. E. Zomkowski, D. Engel, N. H. Gabilan, and A. L. S. Rodrigues, "Involvement of NMDA receptors and 1-argininenitric oxide-cyclic guanosine monophosphate pathway in the antidepressant-like effects of escitalopram in the forced swimming test," European Neuropsychopharmacology, vol. 20, no. 11, pp. 793-801, 2010.

[15] M. Krass, G. Wegener, E. Vasar, and V. Volke, “The antidepressant action of imipramine and venlafaxine involves suppression of nitric oxide synthesis," Behavioural Brain Research, vol. 218, no. 1, pp. 57-63, 2011.

[16] L. Vismari, G. J. Alves, M. N. Muscará, and J. Palermo-Neto, "A possible role to nitric oxide in the anti-inflammatory effects of amitriptyline," Immunopharmacology and Immunotoxicology. In press.

[17] G. Z. Réus, R. B. Stringari, B. De Souza et al., "Harmine and imipramine promote antioxidant activities in prefrontal cortex and hippocampus," Oxidative Medicine and Cellular Longevity, vol. 3, no. 5, pp. 325-331, 2010.

[18] G. Z. Reus, R. B. Stringari, and C. L. Gonçalves, "Administration of harmine and imipramine alters creatine kinase and mitochondrial respiratory chain activities in the rat brain,"
Depression Research and Treatment, vol. 2012, Article ID 987397, 7 pages, 2012.

[19] K. R. Lobato, C. C. Cardoso, R. W. Binfaré et al., “ $\alpha$ Tocopherol administration produces an antidepressant-like effect in predictive animal models of depression," Behavioural Brain Research, vol. 209, no. 2, pp. 249-259, 2010.

[20] S. S. Katyare and R. R. Rajan, "Effect of long-term in vivo treatment with imipramine on the oxidative energy metabolism in rat brain mitochondria," Comparative Biochemistry and Physiology Part C, vol. 112, no. 3, pp. 353-357, 1995.

[21] H. Xu, J. Steven Richardson, and X. M. Li, "Dose-related effects of chronic antidepressants on neuroprotective proteins BDNF, Bcl-2 and Cu/Zn-SOD in rat hippocampus," Neuropsychopharmacology, vol. 28, no. 1, pp. 53-62, 2003.

[22] I. Inkielewicz-Stêpniak, "Impact of fluoxetine on liver damage in rats," Pharmacological Reports, vol. 63, no. 2, pp. 441-447, 2011.

[23] S. Novio, M. J. Núñez, G. Amigo, and M. Freire-Garabal, "Effects of fluoxetine on the oxidative status of peripheral blood leucocytes of restraint-stressed mice," Basic \& Clinical Pharmacology \& Toxicology, vol. 109, no. 5, pp. 365-371, 2011.

[24] A. Zafir, A. Ara, and N. Banu, "In vivo antioxidant status: a putative target of antidepressant action," Progress in NeuroPsychopharmacology and Biological Psychiatry, vol. 33, no. 2, pp. 220-228, 2009.

[25] J. Djordjevic, A. Djordjevic, M. Adzic, I. Elaković, G. Matić, and M. B. Radojcic, "Fluoxetine affects antioxidant system and promotes apoptotic signaling in Wistar rat liver," European Journal of Pharmacology, vol. 659, no. 1, pp. 61-66, 2011.

[26] B. A. Abdel-Wahab and R. H. Salama, "Venlafaxine protects against stress-induced oxidative DNA damage in hippocampus during antidepressant testing in mice," Pharmacology Biochemistry and Behavior, vol. 100, no. 1, pp. 59-65, 2011.

[27] S. D. Khanzode, G. N. Dakhale, S. S. Khanzode, A. Saoji, and R. Palasodkar, "Oxidative damage and major depression: the potential antioxidant action of selective serotonin-re-uptake inhibitors," Redox Report, vol. 8, no. 6, pp. 365-370, 2003.

[28] A. Sarandol, E. Sarandol, S. S. Eker, S. Erdinc, E. Vatansever, and S. Kirli, "Major depressive disorder is accompanied with oxidative stress: short-term antidepressant treatment does not alter oxidative-antioxidative systems," Human Psychopharmacology, vol. 22, no. 2, pp. 67-73, 2007.

[29] H. Herken, A. Gurel, S. Selek et al., "Adenosine deaminase, nitric oxide, superoxide dismutase, and xanthine oxidase in patients with major depression: impact of antidepressant treatment," Archives of Medical Research, vol. 38, no. 2, pp. 247-252, 2007.

[30] M. Bilici, H. Efe, M. A. Köroğlu, H. A. Uydu, M. Bekaroğlu, and O. Deǧer, "Antioxidative enzyme activities and lipid peroxidation in major depression: alterations by antidepressant treatments," Journal of Affective Disorders, vol. 64, no. 1, pp. 43-51, 2001.

[31] J. W. Gawryluk, J. F. Wang, A. C. Andreazza, L. Shao, and L. T. Young, "Decreased levels of glutathione, the major brain antioxidant, in post-mortem prefrontal cortex from patients with psychiatric disorders," International Journal of Neuropsychopharmacology, vol. 14, no. 1, pp. 123-130, 2011.

[32] B. E. Cumurcu, H. Ozyurt, I. Etikan, S. Demir, and R. Karlidag, "Total antioxidant capacity and total oxidant status in patients with major depression: impact of antidepressant treatment," Psychiatry and Clinical Neurosciences, vol. 63, no. 5, pp. 639$645,2009$. 
[33] V. O. Kotan, E. Sarandol, E. Kirhan, G. Ozkaya, and S. Kirli, "Effects of long-term antidepressant treatment on oxidative status in major depressive disorder: a 24-week follow-up study," Progress in Neuro-Psychopharmacology and Biological Psychiatry, vol. 35, no. 5, pp. 1284-1290, 2011.

[34] M. Maes, I. Mihaylova, M. Kubera, M. Uytterhoeven, N. Vrydags, and E. Bosmans, "Lower plasma Coenzyme Q10 in depression: a marker for treatment resistance and chronic fatigue in depression and a risk factor to cardiovascular disorder in that illness," Neuroendocrinology Letters, vol. 30, no. 4, pp. 462-469, 2009.

[35] T. M. Michel, S. Camara, T. Tatschner et al., "Increased xanthine oxidase in the thalamus and putamen in depression," World Journal of Biological Psychiatry, vol. 11, no. 2, pp. 314$320,2010$.

[36] J. W. Gawryluk, J. F. Wang, A. C. Andreazza, L. Shao, L. N. Yatham, and L. T. Young, "Prefrontal cortex glutathione S-transferase levels in patients with bipolar disorder, major depression and schizophrenia," The International Journal of Neuropsychopharmacology, vol. 14, no. 8, pp. 1069-1074, 2011.

[37] B. Halliwell, "Free radicals and antioxidants-quo vadis?" Trends in Pharmacological Sciences, vol. 32, no. 3, pp. 125-130, 2011.

[38] D. R. Janero, "Malondialdehyde and thiobarbituric acidreactivity as diagnostic indices of lipid peroxidation and peroxidative tissue injury," Free Radical Biology and Medicine, vol. 9, no. 6, pp. 515-540, 1990.

[39] D. Del Rio, A. J. Stewart, and N. Pellegrini, "A review of recent studies on malondialdehyde as toxic molecule and biological marker of oxidative stress," Nutrition, Metabolism and Cardiovascular Diseases, vol. 15, no. 4, pp. 316-328, 2005.

[40] N. Zarkovic, "4-Hydroxynonenal as a bioactive marker of pathophysiological processes," Molecular Aspects of Medicine, vol. 24, no. 4-5, pp. 281-291, 2003.

[41] B. Halliwell and C. Y. J. Lee, "Using isoprostanes as biomarkers of oxidative stress: some rarely considered issues," Antioxidants and Redox Signaling, vol. 13, no. 2, pp. 145-156, 2010.

[42] I. Dalle-Donne, R. Rossi, D. Giustarini, A. Milzani, and R. Colombo, "Protein carbonyl groups as biomarkers of oxidative stress," Clinica Chimica Acta, vol. 329, no. 1-2, pp. 23-38, 2003.

[43] G. L. Ellman, "Tissue sulfhydryl groups," Archives of Biochemistry and Biophysics, vol. 82, no. 1, pp. 70-77, 1959.

[44] I. S. Young, "Measurement of total antioxidant capacity," Journal of Clinical Pathology, vol. 54, no. 5, p. 339, 2001.

[45] J. T. Coyle and P. Puttfarcken, "Oxidative stress, glutamate, and neurodegenerative disorders," Science, vol. 262, no. 5134, pp. 689-695, 1993.

[46] A. Gardner and R. G. Boles, "Beyond the serotonin hypothesis: mitochondria, inflammation and neurodegeneration in major depression and affective spectrum disorders," Progress in Neuro-Psychopharmacology and Biological Psychiatry, vol. 35, no. 3, pp. 730-743, 2011.

[47] K. Chopra, B. Kumar, and A. Kuhad, "Pathobiological targets of depression," Expert Opinion on Therapeutic Targets, vol. 15, no. 4, pp. 379-400, 2011.

[48] P. Gałecki, J. Szemraj, M. Bieńkiewicz, A. Florkowski, and E. Gałecka, "Lipid peroxidation and antioxidant protection in patients during acute depressive episodes and in remission after fluoxetine treatment," Pharmacological Reports, vol. 61, no. 3, pp. 436-447, 2009.

[49] R. Harrison, "Physiological roles of xanthine oxidoreductase," Drug Metabolism Reviews, vol. 36, no. 2, pp. 363-375, 2004.
[50] N. Dimopoulos, C. Piperi, V. Psarra, R. W. Lea, and A. Kalofoutis, "Increased plasma levels of 8-iso-PGF $2 \alpha$ and IL-6 in an elderly population with depression," Psychiatry Research, vol. 161, no. 1, pp. 59-66, 2008.

[51] D. Praticò, J. Rokach, J. Lawson, and G. A. FitzGerald, "F2isoprostanes as indices of lipid peroxidation in inflammatory diseases," Chemistry and Physics of Lipids, vol. 128, no. 1-2, pp. 165-171, 2004.

[52] M. Maes, I. Mihaylova, M. Kubera, M. Uytterhoeven, N. Vrydags, and E. Bosmans, "Increased 8-hydroxy-deoxyguanosine, a marker of oxidative damage to DNA, in major depression and myalgic encephalomyelitis/chronic fatigue syndrome," Neuroendocrinology Letters, vol. 30, no. 6, pp. 715722, 2009.

[53] G. Z. Reus, R. B. Stringari, G. T. Rezin et al., "Administration of memantine and imipramine alters mitochondrial respiratory chain and creatine kinase activities in rat brain," Journal of Neural Transmission, vol. 119, no. 4, pp. 481-491, 2012.

[54] P. Rojas, N. Serrano-García, O. N. Medina-Campos, J. Pedraza-Chaverri, S. O. Ögren, and C. Rojas, "Antidepressantlike effect of a Ginkgo biloba extract (EGb761) in the mouse forced swimming test: role of oxidative stress," Neurochemistry International, vol. 59, no. 5, pp. 628-638, 2011.

[55] Z. Zhao, W. Wang, H. Guo, and D. Zhou, "Antidepressantlike effect of liquiritin from Glycyrrhiza uralensis in chronic variable stress induced depression model rats," Behavioural Brain Research, vol. 194, no. 1, pp. 108-113, 2008.

[56] T. Satoh, K. Ishige, and Y. Sagara, "Protective effects on neuronal cells of mouse afforded by ebselen against oxidative stress at multiple steps," Neuroscience Letters, vol. 371, no. 1, pp. 1-5, 2004.

[57] T. Posser, M. P. Kaster, S. C. Baraúna, J. B. T. Rocha, A. L. S. Rodrigues, and R. B. Leal, "Antidepressant-like effect of the organoselenium compound ebselen in mice: evidence for the involvement of the monoaminergic system," European Journal of Pharmacology, vol. 602, no. 1, pp. 85-91, 2009.

[58] C. A. Stockmeier, G. J. Mahajan, L. C. Konick et al., "Cellular changes in the postmortem hippocampus in major depression,” Biological Psychiatry, vol. 56, no. 9, pp. 640-650, 2004.

[59] K. Zou, W. Deng, T. Li et al., "Changes of brain morphometry in first-episode, drug-Naïve, non-late-life adult patients with major depression: an optimized voxel-based morphometry study," Biological Psychiatry, vol. 67, no. 2, pp. 186-188, 2010.

[60] S. Campbell and G. MacQueen, "An update on regional brain volume differences associated with mood disorders," Current Opinion in Psychiatry, vol. 19, no. 1, pp. 25-33, 2006.

[61] A. Kumar, W. Bilker, Z. Jin, and J. Udupa, "Atrophy and high intensity lesions: complementary neurobiological mechanisms in late-life major depression," Neuropsychopharmacology, vol. 22, no. 3, pp. 264-274, 2000.

[62] P. J. Olesen, D. R. Gustafson, M. Simoni et al., "Temporal lobe atrophy and white matter lesions are related to major depression over 5 years in the elderly," Neuropsychopharmacology, vol. 35, no. 13, pp. 2638-2645, 2010.

[63] M. Maes, P. Ruckoanich, Y. S. Chang, N. Mahanonda, and M. Berk, "Multiple aberrations in shared inflammatory and oxidative \& nitrosative stress (IO\&NS) pathways explain the co-association of depression and cardiovascular disorder (CVD), and the increased risk for CVD and due mortality in depressed patients," Progress in Neuro-Psychopharmacology and Biological Psychiatry, vol. 35, no. 3, pp. 769-783, 2011. 
[64] M. Maes, P. Galecki, Y. S. Chang, and M. Berk, "A review on the oxidative and nitrosative stress (O\&NS) pathways in major depression and their possible contribution to the (neuro)degenerative processes in that illness," Progress in Neuro-Psychopharmacology and Biological Psychiatry, vol. 35, no. 3, pp. 676-692, 2011.

[65] J. R. Teyssier, S. Ragot, J. C. Chauvet-Gélinier, B. Trojak, and B. Bonin, "Expression of oxidative stress-response genes is not activated in the prefrontal cortex of patients with depressive disorder," Psychiatry Research, vol. 186, no. 2-3, pp. 244-247, 2011.

[66] B. N. Frey, G. B. Zunta-Soares, S. C. Caetano et al., "Illness duration and total brain gray matter in bipolar disorder: evidence for neurodegeneration?" European Neuropsychopharmacology, vol. 18, no. 10, pp. 717-722, 2008.

[67] L. Minuzzi, G. A. Behr, J. C. F. Moreira, and B. N. Frey, "Mitochondrial dysfunction in bipolar disorder: lessons from brain imaging and molecular markers," Revista Colombiana de Psiquiatría, vol. 40, pp. 166-182, 2011.

[68] F. Kapczinski, F. Dal-Pizzol, A. L. Teixeira et al., "A systemic toxicity index developed to assess peripheral changes in mood episodes," Molecular Psychiatry, vol. 15, no. 8, pp. 784-786, 2010. 


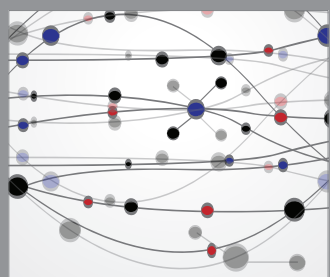

The Scientific World Journal
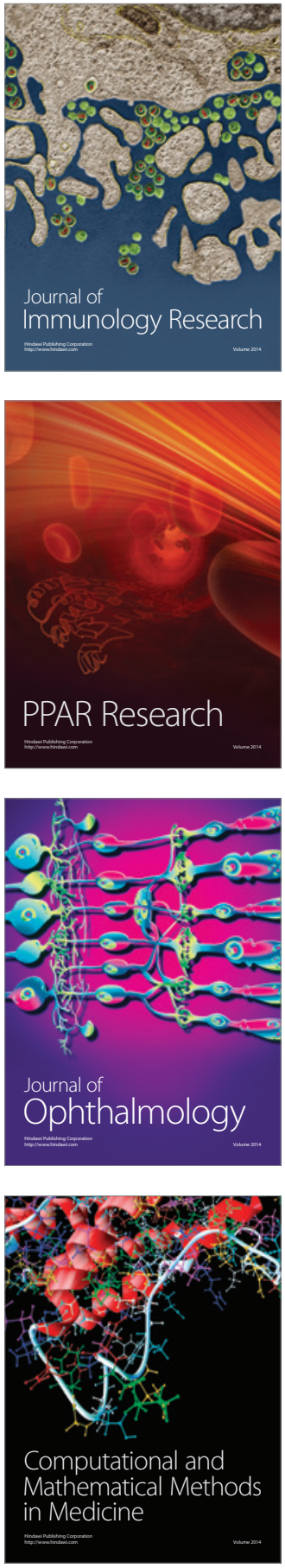

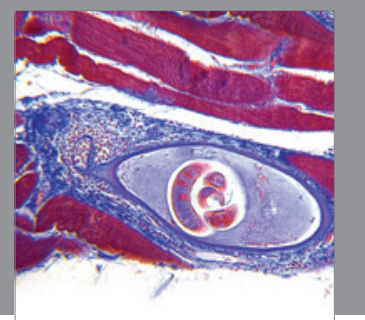

Gastroenterology

Research and Practice
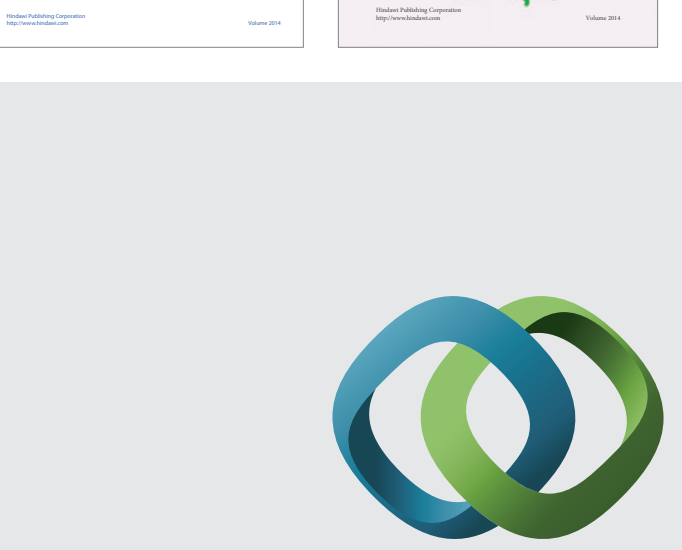

\section{Hindawi}

Submit your manuscripts at

http://www.hindawi.com
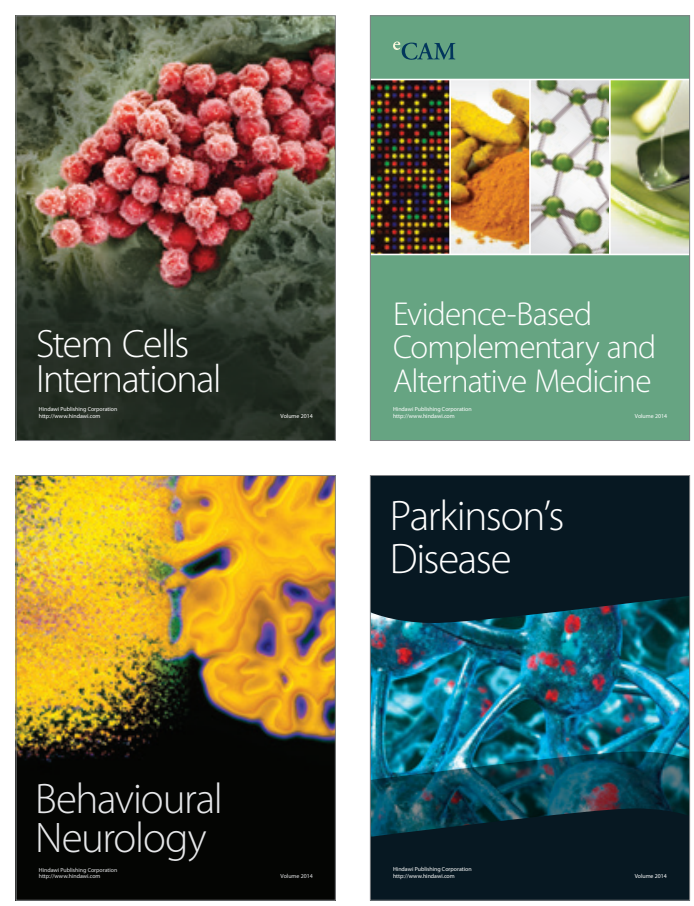

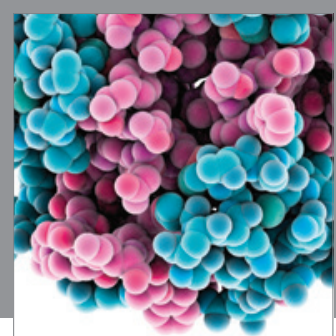

Journal of
Diabetes Research

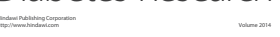

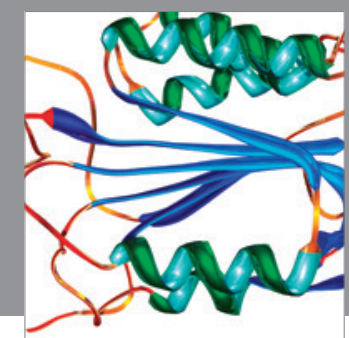

Disease Markers
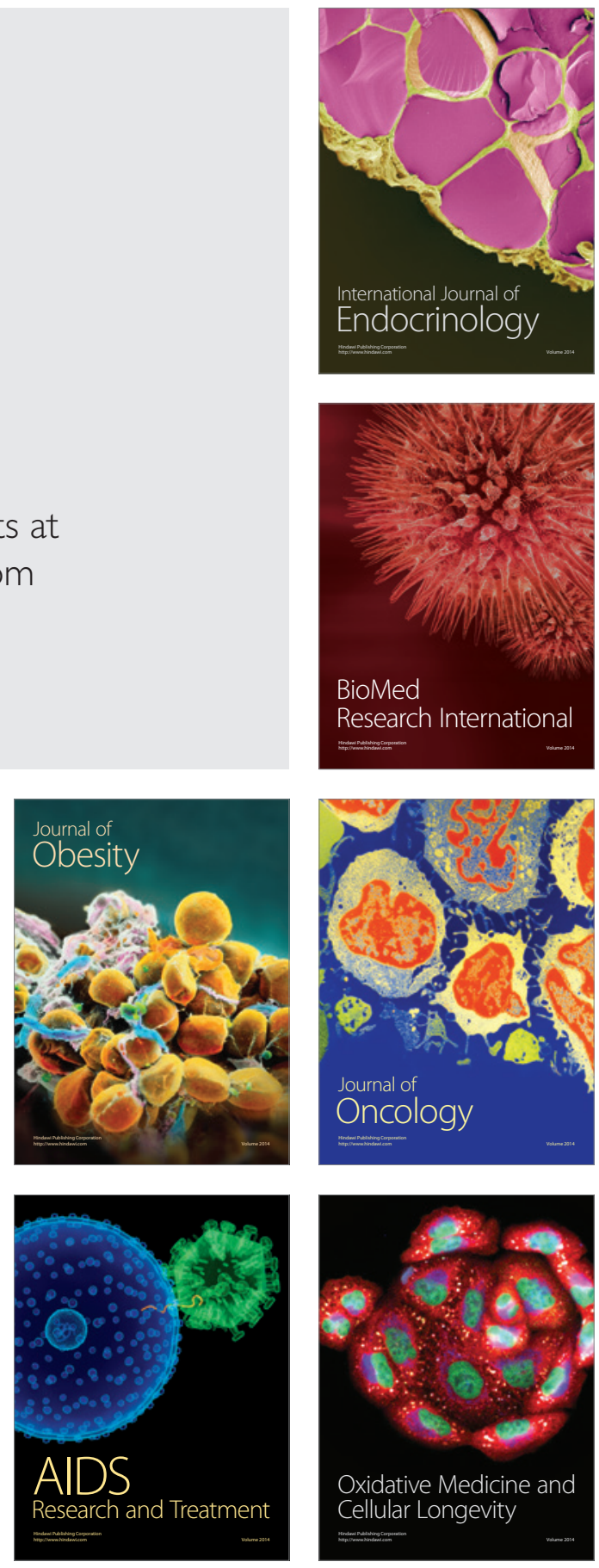